Research Article

\title{
Screening of Gestational Diabetes and its correlation with Maternal and Prenatal Morbidity
}

\author{
Dash Bachaspati*
}

\author{
Associate Professor, Department of Obstetrics and Gynaecology, Shri Shankaracharya Institute of \\ Medical Sciences, Bhilai-490020, Chhattisgarh, India \\ *Corresponding Author: Dash Bachaspati* \\ Address for Correspondance: Dr. Bachaspati Dash \\ Bungalow No.- 36, Royal Greens, Junwani, Bhilai-490020, Chhattisgarh, India
}

\begin{abstract}
:
Introduction: Gestational Diabetes Mellitus (GDM) has public health importance far beyond its immediate effect on the mother and child. Pregnancy is a diabetogenic state and GDM is a major driver of the Type 2 diabetes epidemic. About 1\% - 3\% of all pregnant women will show glucose intolerance. GDM is the most common metabolic complication that affects pregnant women. The frequency of GDM and its associated maternal, prenatal and long term morbidity emphasizes the importance of screening method.

Aim: To study the role of $\mathbf{5 0} \mathbf{g m}$ glucose challenge test for screening of gestational diabetes

Method: Two hundred and fifty antenatal patients between 24-28 weeks of gestation were screened for gestational diabetes by $50 \mathrm{gm}$ oral glucose challenge test. Comparison was made among normal patients and patients diagnosed with gestational diabetes regarding risk factors, clinical examination, routine and special investigations and pregnancy outcome

Results: Out of 250 patients 150 had no risk factors while 100 had one or more risk factors. 10 patients $(4 \%)$ were diagnosed as having gestational diabetes. 8 patients were detected at 24-28 weeks and 2 were detected from the risk factor group at 32-34 weeks.

Conclusion: Universal screening of all pregnant patients during 24-28 weeks with glucose challenge test is simple, convenient, cheap and easy to organize instrument to identify women with GDM. In India we recommend universal screening of all pregnant women, as they have 11 fold increased risk of developing glucose intolerance during pregnancy compared to Caucasian women.
\end{abstract}

Keywords: Gestational Diabetes Mellitus, Glucose Tolerance Test, Maternal Morbidity

\section{NTRODUCTION}

Pregnancy is a diabetogenic state and results when the beta cells are unable to adapt to the Diabetogenic milieu of

pregnancy. Diabetes in pregnancy has been the result of serious consequences to the baby as well as the mother if early detection and appropriate treatment is not done. About $1 \%-3 \%$ of all pregnant women will show glucose intolerance. In India we recommend universal screening of all pregnant women, as they have 11 fold increased risk of developing glucose intolerance during pregnancy compared to Caucasian women ${ }^{1}$. The largest number of these patients is comprised of individualswith geneti or metabolic predisposition towards diabetes and who are incapable of compensating adequately for the diabetogenic effects of pregnancy i.e. patients with gestational diabetes mellitus.
GDM is the most common metabolic complication that affects pregnant women. The frequency of GDM and its associated maternal, prenatal and long term morbidity emphasizes the importance of screening method.

The pregnant diabetic is confronted with health risks. it can result in acute maternal complications like hypoglycemia, ketoacidosis, pre-eclampsia, uti, polyhydramnios, emotional stress and severe maternal complications like preterm labor, diabetic ketoacidosis, nephropathy and retinopathy2,3.

The rationale for using Glucose Challenge Test (GCT) for screening of GDM is based on the physiological changes in normal pregnancy. There is a $10 \%$ reduction in maternal fasting glucose levels by the end of first trimester. The diabetogenic stress of pregnancy is encountered during late gestation and is best recognized in fed state. 
Dash Bachaspati / Screening of Gestational Diabetes and its correlation with Maternal and Prenatal Morbidity

\section{MATERIALS AND METHODS}

The present study was conducted in the antenatal clinic of HiTech Medical College and Hospital Bhubaneshwar after approval from Institutional Ethics Committee. All cases between 24-28 weeks of gestation were screened for GDM by $50 \mathrm{gm}$ glucose challenge test (GCT). Selection criteria included normal pregnant women patients and high risk pregnancy historical or clinical risk factors like, family history of diabetes in first degree relatives, previous delivery of large infants (birth weight $>4 \mathrm{~kg}$ ), poor obstetrics history, presence of a congenital anomaly in a previous child such as neural tube defects, cardiac anomalies, etc, obesity, maternal age over 30 years, glycosuria, polyhydramnios, early onset pre-eclampsia. Patients with diagnosed Type I or II diabetes, hepatic disorders, medications like corticosteroids or insulin sensitizing agents, adrenal tumours or Cushing's disease were excluded. Plasma glucose level of $130 \mathrm{mg} / \mathrm{dl} \quad(7.2$ mmol/l) or higher was considered as positive GCT test ${ }^{4}$. If the GCT was negative it was repeated at $32-34$ weeks especially in obese, elderly and women with risk of GDM. 3 hour $-100 \mathrm{gm}$ oral glucose tolerance test (OGTT) was done on GCT positive patients. GDM was diagnosed when at least two plasma glucose values met or exceeded following values - (NDDG \& ACOG Criteria) Fasting - $105 \mathrm{mg} / \mathrm{dl}$ (5.9 mmol/L),1-hour - 190 $\mathrm{mg} / \mathrm{dl}$ (10.6 mmol /L), 2-hour $-165 \mathrm{mg} / \mathrm{dl}(9.2 \mathrm{mmol} / \mathrm{L})$, 3-hour $-145 \mathrm{mg} / \mathrm{dl}(8.1 \mathrm{mmol} / \mathrm{L})$ Assay of plasma glucose was performed by glucose oxidase - peroxidase method. Data was compiled using chi-square test and Fisher's exact test with a two tailed $\mathrm{P}$ value $<0.05$ being considered significant.

\section{RESULTS}

Out of 250 patients screened at 24-28 weeks 8 patients (3.2\%) were found having positive oral GCT and OGTT. Of these 8 positive patients, 3 patients belonged to Group I i.e. the control group while 5 patients belonged to Group II having one or more risk factors.

Table 1: Shows the results of screening at 24-28 \& 3234 weeks of gestation

\begin{tabular}{|l|c|c|c|}
\hline \multirow{2}{*}{ NUMBER OF PATIENTS } & TOTAL & GROUP I & GROUP II \\
\cline { 2 - 4 } & 250 & 150 & 100 \\
\hline $\begin{array}{l}\text { GCT +VE, OGTT +VE } \\
\text { (AT 24-28 WEEKS) }\end{array}$ & 8 & 3 & 5 \\
\hline $\begin{array}{l}\text { GCT +VE, OGTT +VE } \\
\text { (AT 32-34WEEKS ) }\end{array}$ & 2 & 0 & 2 \\
\hline GDM +VE PATIENTS & 10 & 3 & 7 \\
\hline
\end{tabular}

Of the 250 patients screened 10 patients were diagnosed as having gestational diabetes mellitus out of which 7 had one or more risk factors while 3 patients having no risk factors were found to be having gestational diabetes by the above method of screening. These 10 patients were further studied and followed up till the outcome of their pregnancies.

Table 2: Showing the number of patients who were screened with GCT

\begin{tabular}{|c|c|c|c|c|}
\hline \multirow{2}{*}{$\begin{array}{c}\text { Period of } \\
\text { Gestation }\end{array}$} & \multicolumn{2}{|c|}{$\begin{array}{c}\text { Glucose Challenge } \\
\text { Test }\end{array}$} & \multicolumn{2}{c|}{$\begin{array}{c}\text { Oral Glucose } \\
\text { Tolerance Test }\end{array}$} \\
\cline { 2 - 5 } & Positive & Negative & Positive & Negative \\
\hline $\begin{array}{c}24-28 \\
\text { weeks }\end{array}$ & 50 & 200 & 8 & 42 \\
\hline $\begin{array}{c}32-34 \\
\text { weeks }\end{array}$ & 2 & - & 2 & - \\
\hline
\end{tabular}

Of the 250 patients screened 50 patients had blood sugar values more than or equal to $130 \mathrm{gm} / \mathrm{dl}$ by GCT and out of these 8 patients had OGTT positive at 24-28 weeks. Out of the remaining 42 patients who were GCT positive but OGTT negative, those patients having one or more risk factor were screened at 32-34 weeks again and two of these patients had both GCT and OGTT positive at this gestation. Thus from the above data sensitivity and specificity of GCT came out to be $100 \%$ and $83 \%$ respectively (as the number of false positive patients was 42). In the further study comparison was done between normal pregnancies and pregnancy with GDM as regards risk factors, pregnancy outcome and neonatal morbidity. Maximum number of cases of GDM occurred in the age group of $>30 \mathrm{yrs}$. GDM was seen to be significantly less common $(\mathrm{P}<0.01)$ below 25 yrs of age.

In the normal pregnancy group maximum patients were second gravida (30.62\%) whereas in gestational diabetes positive patient group maximum subjects were fourth gravida and grand multigravida $(30 \%)(\mathrm{P}<0.001)$. The incidence of GDM was significantly lower amongst primigravida and second gravida $(\mathrm{P}<0.001)$. Maximum number of diabetic pregnancies were above $50 \mathrm{~kg}$ weight $(90 \%)$ whereas in the non-diabetic group maximum patients were between $40-59 \mathrm{~kg}$ weight i.e. $63.75 \%$ $(\mathrm{P}<0.001)$. In the normal pregnancy group a positive family history was present in $10.42 \%$ but in GDM group it was $20 \%$ - the difference being highly significant $(\mathrm{P}<0.001)$. In the normal pregnancy group the incidence of preeclamptic toxaemia, polyhydramnios, urinary tract infections, vaginal infections (candidiasis) was 10.63\%, 2.08\%. $6.67 \%$ and $7.9 \%$ respectively, while in the gestational diabetes group these were $20 \%, 10 \%, 20 \%$ and $30 \%$ respectively - the difference between all being highly significant $(\mathrm{P}<$ $0.001)$. One case $(10 \%)$ out of the 10 diabetic patients was controlled by dietary modifications alone while in nine patients $(90 \%)$ human insulin had to be added, the dose varying with the degree of hyperglycaemia.

Preterm labour was observed in 25 patients out of which 23 belong to normal pregnancy group and 2 to GDM group thus constituting an incidence of $9.5 \%$ and $20 \%$ respectively 
which was highly significant $(\mathrm{P}<0.001)$. In the normal pregnancy group the caesarean section rate was $32.5 \%$ as compared to $80 \%$ in the group with gestational diabetes. The difference was highly significant $(\mathrm{P}<$ 0.001).Out of total 86 cases of caesarean section 3 were performed before 37 completed weeks of gestation the cause being pre-eclampsia, malpresentation and fetal macrosomia. Vaginal delivery occurred in 162 patients (67.08\%) of control group as compared to only 2 patients (20\%) of gestational diabetes group. This difference was highly significant.

In the normal pregnancy group the incidence of macrosomia was $3.33 \%$ as compared to $20 \%$ in the pregnancy group with gestational diabetes. This difference was highly significant $(\mathrm{P}<0.001)$. One infant $(5 \%)$ in the diabetic group had major congenital malformation in the form of cardiac anomaly(ASD) and it was premature.A higher incidence of hypoglycaemia (30\%) was found amongst the 3 infants of gestational diabetic mothers and 1 out of these 3 infants was premature.

In the normal pregnancy group hyperbilirubinaemia was present in 3 infants $(1.25 \%)$ of infants, whereas in the infants born to gestational diabetics it was present in 2 infants (20\%), the incidence being significantly $(\mathrm{P}<$ 0.01) high. In the pregnancy group with gestational diabetes two of the infants had RDS (20\%) and one of these was born before 37 weeks of gestation. The incidence of RDS in the normal pregnancy group was $3.75 \%$, (9 infants) of which 7 infants were premature.

The difference in the incidence of RDS in the infants born to normal mothers and gestational diabetics was significant $(\mathrm{P}<0.01)$. Twice weekly nonstress test backed up by foetal biophysical profile were employed for antepartum foetal surveillance of the cases of GDM, in patients with foetal biophysical profile score of 10 there was no perinatal mortality. The perinatal mortality in the normal pregnancy group was $6.67 \%$ but in the gestational diabetic group it was $10 \%$. Prematurity was an associated factor in a most half the cases. The difference between the two groups was significant $(\mathrm{P}<0.05)$.

\section{DISCUSSION}

Pregnancy is a diabetogenic state and GDM has public health importance far beyond its immediate effects on the mother and child. Prevalence of GDM is high in India and diabetes in pregnancy is associated with serious consequences to the baby as well as the mother if early detection and appropriate treatment are not offered. In India, screening is essential universally in all women diagnosed to be pregnant as they have an 11 fold increased risk of developing glucose intolerance during pregnancy compared to Caucasian women. The usual recommendation for screening is between 24 and 28 weeks of gestation.

In this study only 10 patients were found to have gestational diabetes, out of 250 patients enrolled for the study 150 patients did not have any risk factors and were put together in group 1 or control group while 100 patients having one or more risk factors were put in group 2, it was found that gestational diabetes was significantly associated with age group above 30 years and even more significantly with age above 35 years and was less common below 25 years of age 5 which was similar to Abell (1976) who stated that age above 35 years was associated with an increased detection rate of gestational diabetes. Maximum incidence $(30 \%)$ of gestational diabetes was amongst multigravida which was significantly higher statistically than the normal pregnancy group and lower among the primary and second gravida. Our study showed that most of the patients having GDM were above $50 \mathrm{~kg}$ weight which was in accordance with study of Norlander et al (1989) who also reported obesity to be significantly $(\mathrm{p}<0.001)$ associated with gestational diabetes.

The incidence of preeclamptic toxaemia, polyhydramnios, urinary tract infections, vaginal infections (candidiasis) was seen to be more in gestational diabetes as compared to normal pregnancy and the difference was found to be significant $^{6}$, Abdulbari bener et al $^{7}$ in 2011 in Qatar studied the outcome of pregnancy complicated by GDM and concluded that there was increased risk of developing maternal and neonatal complications in pregnant females with GDM.

The rate of caesarean section was found to be significantly higher in GDM group as compared to normal pregnancy which was in accordance to the Toronto tri-hospital study which identified GDM pregnancy having a two-fold increased risk of cesarean delivery 8 A higher incidence of cardiac anomalies, prematurity and hypoglycemia and hyper bilirubinaemia were found in infants of GDM mothers which was similar to the study of Hay WW JR who also found that despite advances in perinatal care, infants of diabetic mothers remain at risk for a multitude of physiologic, metabolic,and congenital complications such as preterm birth, macrosomia, asphyxia, respiratory distress, hypoglycemia, hypocalcemia, hyperbilirubinemia, polycythemia and hyperviscosity, hypertrophic cardiomyopathy, and congenital anomalies, particularly of the central nervous system9. Perinatal mortality was also found to be higher in infants of gestational diabetic group10 which was similar to study of Angadi Rajasab Nilofer et alwhoalso concluded that a higher risk of perinatal outcome is present in women with GDM and effective screening could help in preventing future diabetes mellitus in the mother and also in the unborn child.

\section{CONCLUSION}

Gestational diabetes has many maternal and foetal complications so it is necessary to detect gestational diabetes. Screening of all pregnant patients during 2428 weeks with glucose challenge test is simple, convenient, cheap and easy to organize at outpatient setting. Thus we may conclude that universal screening remains a good instrument to identify women with GDM at least in populations like our Indian 


\section{Dash Bachaspati / Screening of Gestational Diabetes and its correlation with Maternal and Prenatal Morbidity}

population where there is high prevalence of diabetes and outcomes .Indian J Endocrinol Metab. Mar 2012;

a large percentage of women are at medium or high risk for GDM. Thereby screening, proper management and follow up of women with GDM and their offspring has the potential to prevent diabetes in two generations.

\section{REFERENCES:}

[1] Dornhost A, Paterson CM, Nicholls JS, Wadsworth J, Chiu DC, Elkeles RS, et al. High prevalence of GDM in women from ethnic minority groups. Diabetic Med. 1992;9:820-2.

[2] Diabetes and endocrine disease in pregnancy. Dewhurst obstetrics and gynecology 7th ed. Dewhurst .pg 248254.oxford.2007.

[3] Schaefer UM etal. Congenital malformations in offspring of women with hyperglycemia first detected during pregnancy.AM J Obstet Gynaecol.1997;177:1165-71.

[4] American college of obstetricians and gynecologist committee on practice bulletins - ACOG practice bulletin. Clinical management guidelines for obstetrician gynaecologists. Number 30, 2001.

[5] Karoline Kragelund Nielsen, Anil Kapur, Peter Damm, Maximilian de Courten, Ib Christian Bygbjerg

[6] BMC Pregnancy Childbirth. 2014; 14: 41. Published online 2014 January 22. doi: 10.1186/1471-2393-14-41

[7] Mamta bhat et al. Study of Gestational Diabetes Females in thiruvananthapuram. MJ J Diabetes Dev Ctries.2010;30(2):91-96.

[8] Abdul Bari et al. Outcome of pregnancy complicated by Gestational Diabetes Mellitus. Int J Women Health, 2011;3:367-373.

[9] Sermer M, Naylor C, Kenshole A, et al. The Toronto TriHospital Gestational Diabetes Project: A preliminary review. Diabetes Care. 1998;21 (Suppl 2):833-842.

[10] Hay WW Jr.Care of infant of a diabetic mother :Curr Diab Rep.2012 Feb;12(1):4-15

[11] Angadi Rajasab Nilofer, V. S. Raju, B. R. Dakshayini, andSyed Ahmed Zaki Screening in high-risk group of gestational diabetes mellitus with its maternal and fetal 16(Supp11): S74-S78. 